\title{
TALLERES PARA DOCENTES: ACTIVIDADES PARA LA ATENCIÓN DE LA DIVERSIDAD ESCOLAR
}

\author{
MEd. Angélica Fontana Hernández ${ }^{1}$
}

\section{RESUMEN}

La atención de todos los estudiantes es un reto que se impone cada día al sistema educativo costarricense y las transformaciones substanciales que requiere se empujan desde sus bases.

La escuela, como institución social, espacio de aproximación de la diversidad existente y responsable de la formación de las futuras generaciones, se enfrenta a la difícil tarea de ofrecer respuestas adecuadas a las diversas capacidades y necesidades de la población estudiantil.

Desde esta perspectiva, se diseña una propuesta que permite a los docentes de la Educación General Básica (preescolar, I y II ciclos, materias especiales y de otra lengua) desarrollar habilidades en la elaboración de estrategias pedagógicas para la atención de la diversidad estudiantil.
La propuesta contempla diez talleres que siguen un proceso de reflexiónparticipación-construcción e intercambio. Los participantes podrán expresar sus ideas, inquietudes y emociones sobre los temas abordados construir conceptos, materiales y estrategias didácticas para la atención de la diversidad.

\section{Palabras claves}

Atención a la diversidad, propuesta metodológica, docentes.

\section{ABSTRACT}

The attention of all students represents a big challenge for the Costa Rican educational system and all significant required transformations are pushed since its foundations.

The school, understood as a social institution, space in which diversity is approached and also responsible for

1 Académica de la Universidad Nacional. Labora como docente en la División de Educación Básica y en el Proyecto UNA Educación de calidad para los estudiantes con discapacidad de la DEB. Máster en Pedagogía con énfasis en diversidad de los Procesos Educativos-UNA. 
the instruction of future generations, is facing the difficult task of providing appropriate answers to the diverse needs of the student population.

From this perspective, a proposal is designed that allows teachers from General Basic Education (pre-school, I and II Cycles, special and foreign language classes) to develop skills in the implementation of pedagogic strategies for the attention of classroom diversity.

The proposal includes ten workshops that follow a reflection-partici- pation-construction and exchange process. Participants will be able to express their ideas, interests and emotions concerning the topics discussed. They will also develop concepts, materials and didactic strategies for the attention of their students

\section{Key Words}

Diversity attention, methodological proposal, teachers.

C

ada mañana, se miran pasar grupos de niños, niñas y jóvenes que van presurosos por llegar a tiempo a sus centros educativos. Algunos llevan sobre sus espaldas grandes bultos y otros abrazan libros y cuadernos contra sus pechos; pero todos guardan en sus corazones la esperanza de alcanzar una formación que les permita una realización personal y social.

La atención de todos los estudiantes es un reto que se impone cada día al sistema educativo costarricense y las transformaciones substanciales que esto requiere, empujan desde sus bases, poco a poco, los cambios requeridos.

El reconocimiento de la diversidad estudiantil en el ámbito escolar es un hecho ineludible, porque cada estudiante coexiste en distintas realidades, estructuras familiares, contextos sociales y culturales donde las diferencias y semejanzas juegan un papel determinante en la convivencia humana.

La escuela, como institución social y espacio de aproximación de la diversidad existente y creciente de la sociedad global (Fernández, 2001) y, principalmente, como responsable de la formación de las futuras generaciones, se enfrenta ante la difícil tarea de ofrecer respuestas adecuadas a las diversas capacidades y necesidades que exige la población estudiantil de hoy y de cumplir con su función socializadora, inculcando las pautas culturales, morales y normativas vigentes.

El reconocimiento de la diversidad como un elemento fundamental en la educación de una nación, requiere de una organización política y social que la fortalezca para que se puedan realizar los cambios necesarios en la organización escolar y en la formación docente. También, urge la concientización de los responsables para efectuar las transformaciones en los sistemas educativos de acuerdo con las exigencias de los grupos sociales que, históricamente, 
han tenido posiciones de desventaja social, tales como indígenas, negros, campesinos, obreros, personas con discapacidad, entre otros y tomando en cuenta las nuevas perspectivas del pensamiento crítico y completo, humanista y constructivista de la pedagogía.

De acuerdo con Meléndez (2002), los esfuerzos realizados en Latinoamérica y en la región centroamericana por democratizar la educación han sido en términos de cobertura, de respeto a algunas formas de identidad (lengua autóctona) y de mejoramiento de la calidad de los servicios educativos, los cuales surgieron por las enormes luchas y sacrificios de las minorías activistas; sin embargo, no satisfacen los acuerdos ratificados por estas naciones en los convenios internacionales sobre derechos humanos, derechos de las y los niños y contra todas las formas de discriminación.

El proceso de formación docente tiene un papel esencial en el desarrollo integral de las y los estudiantes y en la adaptación y aceptación de quienes presentan necesidades educativas. También, influye en la interiorización y la práctica de los valores de la tolerancia, el respeto, la igualdad, la libertad y de los derechos humanos fundamentales en la interacción con sus compañeros, docentes y personal administrativo de la institución educativa.

Para lograr este cambio, la preparación y capacitación docente se constituye en un elemento fundamental, porque el y la docentes son los encargados de desarrollar las destrezas básicas, los valores y las actitudes en las nuevas generaciones. Más que teoría, las universidades deben implementar experiencias o proyectos con grupos representativos de la diversidad, como alumnos marginados por su cultura, desertores, trabajadores de la calle, excluidos del sistema educativo o con necesidades educativas especiales por mencionar algunos. El objetivo primordial es sensibilizarlos y propiciar espacios educativos, que desarrollen sus destrezas profesionales, necesarias para la atención a la diversidad. Al respecto, Zúñiga (2002) menciona: "realmente queremos futuros profesionales que entiendan de diversidad, pero que por encima de todo, puedan en un futuro no muy lejano ofrecer servicios profesionales, públicos, privados, a una sociedad naturalmente diversa" (p. 145).

Si se logra un proceso de concientización hacia la diversidad desde el inicio de su carrera profesional, el impacto en la atención de las diferencias individuales y las necesidades educativas especiales será mayor en cuanto a su calidad y cantidad. La imaginación, la creatividad y la actitud que tenga el profesorado ante este nuevo reto, serán fundamentales. Según López (2002), "esa actitud que tengo hacia la vida de creer en lo qué hago y en cómo lo 
hago, como algo que vale la pena" (p.13) marcará la diferencia en este urgente proceso de transformación social en y para la diversidad.

El currículo para la atención a la diversidad debe responder a los principios de igualdad y equidad educativa, ofreciendo las mismas oportunidades a las y los estudiantes donde las necesidades y capacidades sean respetadas y desarrolladas.

Debe ser abierto, flexible y participativo, que incluya la diversidad lingüística, cultural y personal de cada estudiante, que ofrezca respuestas educativas y niveles de concreción de acuerdo con las exigencias específicas de sus actores y permita en su planificación la adecuación y adaptación de las metodologías y de los recursos, sin encubrir las desigualdades sociales, culturales y económicas. Por lo tanto, cada centro educativo ha de propiciar el desarrollo de un currículo funcional y contextual para que todos los estudiantes puedan expresar sus intenciones y necesidades educativas en los programas y proyectos que se desarrollan. Al respeto, Belmonte (1998) opina que "la comprensividad escolar va asociada a finalidades sociales y políticas... por eso se le atribuye al currículo un importante valor de cambio, en la medida que proporciona las mismas oportunidades para todos los alumnos y compensa las desigualdades sociales y culturales" (p. 16).

Considerando lo anterior, es fundamental que el docente identifique y denuncie las prácticas ocultas del currículo oficial que son obstáculos para aquellos estudiantes diferentes o que se encuentren en una situación de desventaja social, económica o cultural, y que les impide desarrollarse como sujetos activos, críticos y emprendedores. También debe ser capaz de generar experiencias cotidianas posibilitadoras mediante el uso de un lenguaje crítico y reflexivo e incorporar variedad de estrategias didácticas que faciliten la participación de todos los estudiantes sin ningún tipo de discriminación o exclusión y la construcción del conocimiento en forma colectiva. Es decir, debe propiciar una educación social que reconozca la diversidad que presentan los estudiantes y se preocupe por ofrecer alternativas curriculares que les permitan una incorporación sociolaboral. Giroux (1990) menciona que los profesores deben asumir el papel de intelectuales transformadores que emprendan una enseñanza como práctica emancipadora y se comprometan con la creación de comunidades escolares que vivencian los principios y los valores democráticos de la igualdad, equidad y justicia social.

La enseñanza, al estar cargada de intencionalidad (López, 2002), podrá formar una nueva cultura caracterizada por los valores de la diversidad, tales como la cooperación, la tolerancia, el respeto a las diferencias, la solidaridad, el amor, la igualdad, la justicia y la equidad para todos. 
Desde la concepción constructivista, la o el docente tiene la función de ser un guía, un orientador, un facilitador para que cada estudiante pueda construir sus conocimientos partiendo de sus habilidades cognitivas, de los conceptos previos, de la capacidad de reflexionar, analizar y de las oportunidades de explorar, descubrir, ensayar y aproximarse al contenido estudiado. Lo anterior pone de manifiesto la importancia de establecer una relación interpersonal en los procesos de enseñanza y de aprendizaje donde el docente ajusta su intervención pedagógica, tomando en cuenta el nivel de desarrollo de su estudiante, sus capacidades y necesidades para alcanzar una mayor compresión, es decir, un aprendizaje escolar.

De acuerdo con Pérez (1994), la finalidad de la intervención pedagógica es "contribuir a que el alumno desarrolle la capacidad de realizar aprendizajes significativos por sí mismo en una amplia gama de situaciones y circunstancias, es decir, que el alumno aprenda a aprender" (p. 179). Menciona, además, que entre las condiciones que favorecen el aprendizaje significativo se encuentran la presentación de conflictos para que los esțudiantes resuelvan y confronten puntos de vista diferentes en la solución del problema, el análisis del error y del proceso vivido en el logro del resultado o del producto. Por lo anterior, en el aprendizaje significativo se privilegia el proceso construido, las competencias específicas y no el objetivo final.

El reconocimiento de la diversidad como valor implica diferentes etapas. Una de ellas es la tolerancia del otro, entendida como las actitudes positivas hacia las diferencias físicas, sociales y culturales de cada individuo. El respeto es el siguiente paso, que es el reconocimiento de las diferencias del otro en la interacción cotidiana, sin buscar la aculturación o eliminación de estas. Y por último, la aceptación es el paso más difícil, porque implica ir más allá de las relaciones humanas; significa tener una apertura para aprender del otro y compartir lo que se tiene en común, es decir, convivir. Por eso, el reconocimiento a la diversidad debe convertirse en el aspecto fundamental de la intervención pedagógica para evitar las desigualdades y desventajas en el contexto escolar.

Es importante resaltar que la diversidad de características que presentan los estudiantes, puede convertirse en un aspecto valioso para los procesos de enseñanza y de aprendizaje, si la y el docente las consideran como variables que fortalecen y dinamizan los canales de aprehensión de cada estudiante y planifica en forma estratégica su aprovechamiento en las diversas actividades que se realizan en el aula y en el contexto escolar. De acuerdo con Meléndez (2002), "la diversidad tiene la capacidad de multivariar los recursos para la 
solución interactiva de problemas, reconociendo una extensión del conocimiento propio en el conocimiento aprendido de la diversidad de estilos que encuentra entre sus compañeros" (p. 39).

Atender las diferencias individuales de cada estudiante no es tarea fácil para el personal docente y administrativo de un centro educativo; más difícil es lograr que estas diferencias enriquezcan los procesos de enseñanza y de aprendizaje de todos los actores. Las aulas están formadas por diversidad de estudiantes que tienen distintos orígenes culturales y lingüísticos, diversos talentos y capacidades, necesidades educativas derivadas o no de una discapacidad y estas diferencias pueden generar exigencias en el área académica, emocional y social, las cuales merecen ser atendidas con una educación de calidad.

Ante esta diversidad de características, la y el docente enfrentan un desafio profesional: preparan lecciones accesibles para todos sus alumnos, es decir, crear estrategias para la diversidad estudiantil y que procuren el éxito de cada uno sin encubrir las desigualdades sociales, económicas y culturales.

El juego educativo es una herramienta eficaz en el aprendizaje, porque facilita la construcción y comunicación del conocimiento, aspecto esencial para el abordaje pedagógico desde una perspectiva constructivita social. De acuerdo con Gómez (citada por Sánchez, Flores y Céspedes, 2001), en los diversos espacios de aprendizaje que contiene el contexto escolar, los juegos educativos constituyen un apoyo valioso para el docente, puesto que despiertan el interés de los estudiantes por ciertos temas, se pueden trabajar en forma grupal e individual y se encuentran diferentes tipos de juegos (simbólicos, tradicionales, organizados y predeportivos), los cuales se utilizan según el objetivo pedagógico abordado.

Asimismo, el juego con reglas permite a los participantes negociar formas de comportamiento adecuadas en el desarrollo de las actividades. Es importante resaltar que estas habilidades son necesarias en la interacción, la comunicación y el aprendizaje significativo; además, la actividad lúdica con fines didácticos amplía las posibilidades de acción, imaginación y creación de conocimientos en ausencia de ambientes reales que los generen.

De acuerdo con Belmonte (1998), educar en la diversidad implica acoger un modelo de desarrollo curricular que posibilite el aprendizaje a cada estudiante, según su diversidad personal, familiar y social, siendo más una convicción cultural que un recurso técnico.

Este autor propone los siguientes principios pedagógicos para dar respuesta a la diversidad: 
Originalidad frente al alumno tipo: se refiere de las diferencias individuales como fuente de enriquecimiento de la acción educativa.

Heterogeneidad frente a homogeneidad: consiste en la agrupación flexible de los estudiantes dentro de un marco de respeto y tolerancia en una sociedad plural y democrática.

Coeducación frente a sexismo: se relaciona con el trabajo escolar en igualdad de derechos, deberes y oportunidades entre ambos sexos reconociendo y aceptando las diferencias.

Interculturalidad frente a monolitismo y etnocentrismo cultural: se refiere al espacio de diálogo y comprensión entre estudiantes o colectivos con características culturales distintas en un ambiente de respeto y tolerancia mutuos.

Identificación de objetivos generales: consiste en la definición de un marco común de objetivos y experiencias generadoras de aprendizajes, utilizando variedad de recursos y estrategias para potenciar al máximo las capacidades de todos los (as) estudiantes.

Acción positiva a favor de los más desfavorecidos: consiste en realizar todos los esfuerzos pedagógicos para tratar de compensar las desigualdades de los y las estudiantes y favorecer la igualdad de oportunidades proporcionando diversos apoyos.

Relatividad, temporalidad e interactividad de las necesidades educativas: se refiere a que las necesidades educativas están relacionadas con un contexto determinado y pueden variar durante el tiempo, según la interacción familiar, escolar y comunal, pudiendo aminorarse, modificarse, atenuarse, compensarse de acuerdo con la evolución intelectual, psicológica y emocional.

Es de vital importancia que el docente se preocupe por considerar estos principios pedagégicos en la selección de las estrategias de enseñanza, los recursos didácticos y en las formas de evaluación en la atención individual que se les proporciona a los y las estudiantes, principalmente, aquellos que presentan necesidades educativas especiales.

El nuevo paradigma biosicosocial reconoce la influencia del contexto en la acción educativa, dejando atrás el énfasis en las limitaciones y considerando las necesidades educativas como una condición más que presentan los y las estudiantes en su proceso de formación, como son los recursos didácticos, el estilo de aprendizaje, los métodos de enseñanza, la cultura, entre otros. Según Zúñiga (2002), "Al asumir este carácter interactivo las necesidades educativas de los alumnos tienen entonces un carácter relativo" (p. 144), ei cual debe ser abordado en el proceso educativo, especialmente en 
la determinación de las estrategias más apropiadas para atender dichas necesidades y realizar las adaptaciones específicas para el respeto y atención de la diversidad.

De acuerdo con Brennan (citado por Aguilar, 2000), las necesidades educativas especiales se definen como aquellas dificultades mayores que posee una alumna o un alumno para acceder a los aprendizajes comunes para su edad, ya sea por causas internas (aspectos endógenos) o por un planteamiento educativo inadecuado (aspectos exógenos), que requieren de unas condiciones y adaptaciones en el aprendizaje para compensar dichas dificultades y la provisión de recursos específicos.

Resulta fundamental que las y los docentes reconozcan que sus estudiantes presentan diversidad de características generadas de sus diferencias individuales, contextuales y socioculturales, así como necesidades educativas derivadas o no de una discapacidad y que estas requieren ser abordadas no como excepción, sino como parte de la normalidad diferenciada que se presenta cotidianamente en distintos contextos, en especial en el aula escolar.

Además, eso hace necesario que cada docente comprenda que la identificación oportuna de las habilidades, destrezas, inteligencias, talentos o necesidades educativas le va a permitir proporcionar una atención pedagógica adecuada, elaborar programaciones individuales, diversificar las estrategias de enseñanza, adaptar los materiales didácticos y recursos, ofreciendo oportunidades de construcción del conocimiento, que favorecen un desarrollo armonioso de cada estudiante, independientemente de sus cualidades y distinciones.

Para lograr atender estas necesidades educativas especiales, desde un enfoque de la diversidad, se necesita de un proceso diagnóstico, tanto del estudiante como del contexto escolar, familiar y comunal para determinar las habilidades y necesidades en cada ámbito; asimismo, se debe emplear variedad de instrumentos y técnicas, como fichas personales de desempeño, observación del o la estudiante en diferentes contextos, entrevistas y diálogos, tanto con el o la estudiante, los padres y’docentes, pruebas escritas, orales y de ejecución, trabajos elaborados por el o la estudiante y pruebas psicometrías.

Con los resultados obtenidos, se articula una respuesta que considera una visión humanista, inclusiva, constructivista y democrática del centro educativo y que permita diversas formas de concreción curricular en el aula. Al respecto, Belmonte (1998) expresa la importancia de conceder a los centros educativos plena autonomía institucional en materia curricular, para que se posibilite, paulatinamente, la introducción de las modificaciones y adaptaciones a las distintas diversidades detectadas en el contexto escolar. 
En otras palabras, se deberán ajustar, en forma natural, las modificaciones en la planificación y la concreción del currículo, seleccionando los objetivos, los contenidos, elaborando materiales, generando o adaptando los métodos y aplicando una evaluación flexible y específica.

Este autor propone los siguientes niveles de concreción curricular:

El primer nivel es el Proyecto Educativo de Centro, en el cual se expresa la posición que tomará la institución en la aceptación y la atención de la diversidad de sus estudiantes.

El segundo nivel es el Proyecto Curricular, la y el docente definirán cuáles objetivos y contenidos enseñar, la distribución de los contenidos en los ciclos y su temporalización, la diversidad de metodologías, la organización del espacio, la agrupación de los y las estudiantes, según sus capacidades y distinciones y las estrategias e instrumentos de valoración y evaluación.

El tercer nivel es la programación de aula, que incluye el conjunto de unidades didácticas que expresan los procesos de enseñanza y de aprendizaje, según las peculiaridades de la población estudiantil.

Es importante resaltar que en cada nivel de concreción curricular, se deben tomar determinaciones que van a repercutir en forma de cascada en cada nivel y es precisamente en la dinámica del aula escolar donde se presentan "el mayor número de decisiones concretas para alumnos concretos y en momentos determinados" (Belmonte, 1998, p. 37) que definirán las adaptaciones específicas para el respeto y atención de la diversidad escolar.

Desde esta perspectiva, se diseña una propuesta que permite a los y las docentes de la Educación General Básica (preescolar, I y II ciclos, materias especiales y de otra lengua) desarrollar habilidades en la elaboración de estrategias pedagógicas para la atención de la diversidad estudiantìl en concordancia con los planteamientos antes expuestos.

La propuesta se desarrolla mediante diez talleres que siguen un proceso de reflexión-participación-construcción e intercambio. Los y las participantes podrán expresar sus ideas, inquietudes y emociones sobre los temas abordados, construir conceptos, materiales y estrategias didácticas para la atención de la diversidad.

Los talleres tienen la siguiente estructura: objetivo específico, número de participantes, materiales y equipo tecnológico, tiempo aproximado y procedimientos que incluyen tanto las actividades realizadas por los y las docentes participantes como por la facilitadora.

Cada taller se inicia con actividades que le permite al o la docente reflexionar y vivenciar los temas abordados desde su experiencia personal, 
familiar, social y pedagógica. La facilitadota realiza un cierre de cada sesión, considerando las construcciones efectuadas por los y las participantes y los aportes teóricos respectivos.

La evaluación se efectúa al final de cada taller y es de tipo formativa. Cada participante puede expresar en los instrumentos los conocimientos aprendidos, los que puede llevar a la práctica y el tipo de apoyo (técnico, material y humano) que requiere, los sentimientos y las emociones generadas durante cada sesión de trabajo y algún comentario o sugerencia para mejorar el taller.

A continuación, se presenta una breve descripción de algunos de los talleres:

El primer taller tiene como objetivo comprender el concepto de diversidad y sus implicaciones en el ámbito personal, familiar, escolar y comunal. Una de las técnicas utilizadas es la Silueta Humana, en la cual sobre una figura que está doblada, la o el docente dibuja al frente su imagen corporal y en el interior, al lado derecho, escribe o expresa en forma gráfica sus características emocionales, cognitivas, espirituales, físicas, culturales y sociales y al lado izquierdo, sus necesidades. Luego, comparte su experiencia personal y construye, en forma individual y grupal, el concepto de diversidad. También, determina, en forma conjunta, el impacto de este concepio en las interrelaciones familiares, escolares y comunales. Posteriormente, la facilitadota realiza un cierre de la sesión de trabajo y cada participante completa el instrumento de evaluación.

El otro taller le permite al docente identificar las habilidades profesionales que demanda el apoyo a la diversidad escolar. Una de las técnicas empleadas es el Espejo de mi práctica pedagógica, en la cual la y el docente pueden reflexionar sobre su labor en la escuela, expresar conceptos relacionados con los procesos de enseñanza y de aprendizaje, las estrategias de mediación y evaluación empleadas, entre otros. Luego, con ayuda de la facilitadora, cada participante contrasta su perfil pedagógico con el que se requiere para la atención de la diversidad escolar. Luego, en forma grupal, se analizan e identifican las habilidades profesionales, intelectuales, emocionales, de comunicación y sociales que demanda la atención educativa de la diversidad escolar. La facilitadora realiza el cierre de la sesión y se completa el instrumento de evaluación.

Otros dos talleres le permiten al o la docente analizar y aplicar diferentes formas de intervención pedagógica que faciliten la atención a la diversidad escolar. Se realizan guías de trabajo en subgrupos de tres a cinco personas 
relacionadas con diferentes técnicas de atención a la diversidad escolar, en la cual las y los docentes pueden construir, adaptar, recrear o enriquecer estás técnicas según su experiencia personal, familiar y social, propiciando de esta forma el intercambio de experiencias pedagógicas.

Un último taller se destina para examinar los aspectos positivos, las oportunidades, las dificultades y las limitaciones que enfrenta el o la docente para llevar a la práctica diferentes estrategias metodológicas para responder a la diversidad estudiantil. Además, se solicita a los participantes expresar en forma literaria, gráfica, rítmica o plástica los conocimientos y destrezas adquiridos durante la realización de los talleres, con el fin de publicar los resultados. 


\section{REFERENCIAS}

Aguilar, L. (2000). De la integración a la inlusividad. La atención a la diversidad pilar básico en la escuela del siglo XXI. Buenos Aires: Espacio Editorial.

Belmonte, M. (1998) Atención a la diversidad I Diseño de estrategias y evaluación formativa basada en el análisis de tareas. Bilbao: Ediciones Mensajero

Fernández, M. (2001). Educar en tiempos inciertos. Madrid: Ediciones Morata.

Giroux, H. (1990) Los profesores como intelectuales. Hacia una pedagogía critica del aprendizaje. Buenos Aires: Ediciones Paidós.

López, M. (2002) Ideología, diversidad y cultura: Una nueva escuela para una nueva civilización. En: Equidad y calidad para atender a la diversidad. Brasil: Editorial Aique

Meléndez, L. (2002) La inclusión escolar del alumno con discapacidad intelectual Bogotá: Creamos alternativas GLARP-IIPP

Pérez, R. (1994). El curriculo y sus componentes. Barcelona: OIKOS-TAU.

Sánchez, M., Flores, L. y Céspedes, R. (2001) Comunicación y lengua escrita: transitando entre el contexto social el texto escrito. San José: Ministerio de Educación Pública, OIM USAID, Universidad Nacional

Zúñiga, E. (2002) Las adecuaciones curriculares en la universidad: un derecho y una responsabilidad impostergables. En: Las personas con discapacidad en la educación superior. San José: Fundación Justicia y Género. 